\title{
"Epistemólogos, metodólogos y científicos: La formación y conversión de científicos a teorías hegemónicas."
}

\author{
Epistemologist, methodologist and scientists: The formation and scientists' conversion \\ to hegemonic theories.
}

Francisco Covarnubias Villa y Ma. Guadalupe Cruz Navarro

Instituto Politécnico Nacional, Centro Interdisciplinario de Investigación para el Desarrollo Integral Regional, Unidad Michoacán, México. pancheco@prodigy.net.mx y lupitacuuz63@hotmail.com

\section{Resumen}

Considerando que la formación epistemológica es esencial para la realización de la práctica cientúfica, en este trabajo, se aborda la manera en la que se realiza el proceso de formación de cientúficos, mostrando las características que diferencian al epistemólogo del metodólogo y de los profesores de los futuros cientúficos, para determinar cómo inciden en la formación de cientúficos en teorías hegemónicas y en la incorporación de cientúficos ya formados a la nueva teoría paradigmática.

\section{Palabras clave}

Formación, científicos, paradigma, teoría, enigmas.

\section{Abstract}

Considering that the epistemology formation is essential in the conscience of a scientist, in this work, is approached the way in the one that is realized the process of scientists' formation, showing characteristics that differentiate to the epistemologist of methodologist and of the teachers of the future scientists, for to determine how they fall in the scientists' formation in hegemonic theories and in the scientists' incorporation already formed to the new paradigmatic theory.

\section{Key words}

Formation, scientists, paradigm, theory, enigmas.

\section{Introducción}

Se considera que la formación de científicos implica la generación de conciencias cuíticas, cultas y reflexivas, que conduzcan a pensar lo impensado y lo posible. Hoy día, entre los científicos no es común encontrar reflexión epistemológica ni el desarrollo de prácticas investigativas sustentadas en un conocimiento filosófico profundo. Evidentemente, este hecho denota una división del quehacer intelectual que conlleva múltiples limitaciones cognitivas en los sujetos que se encuentran en formación. El epistemólogo instruye con una centración en las implicaciones lógicas de la práctica investigadora, el metodólogo en las técnicas y procedimientos a escoger para la construcción de instrumentos que hacen posible la percepción de formas y contenidos espećficos de los objetos de investigación y el especialista en la construcción de conocimiento de algún aspecto de la teoría que ha asumido. La supresión de la reflexión filosófica en la formación de los futuros cientúficos, va acompañada de la especialización cognitiva extrema, ya que en los planes de estudio de los programas de formación profesional se encarna la concepción fragmentarista predominante en la actualidad. Por otra parte, la formación de científicos se da en un marco en el que se impide la formación arítica del 
futuro científico, al exduir el estudio profundo de la historia de la ciencia y de los debates más relevantes en el campo de la filosofía de la ciencia y la epistemología.

\section{Desarrollo}

\section{El quehacer de los metodólogos y los epistemólogos}

No es frearente la reflexión epistemológica entre los científicos, pero tampoco lo es la práctica investigadora de objetos concretos reales entre epistemólogos y metodólogos. La mayoría de los metodólogos se ocupan en el estudio de las diferentes técnicas y procedimientos empleados en una disciplina científica o en disaiplinas científicas cercanas y no en la producción de propuestas generadas en prácticas investigativas de objetos concretos. Algunos llegan a relacionar los bloques tecnoprocedimentales con corrientes de pensamiento teónico, pero casi nunca incorporan la reflexión epistemológica a sus disaursos metodológicos, pues ésta ha sido reservada a los epistemólogos. Generalmente, los epistemólogos son sujetos intelectualmente potentes, pero colocados muy lejos tanto de la reflexión de la dimensión tecno-procedimental de la práctica investigadora, como de la práctica constructora de conocimiento de objetos concretos. Mientras que el epistemólogo asume una postura crítica metadisaiplinaria, el metodólogo quiere colocarse por encima de las posturas teóricas asumiendo consciente o inconscientemente una de ellas erigiéndola en metarracionalidad. En tanto que el filósofo construye entramados con las categorías y conceptos generados en las diferentes prácticas cientúficas para construir discursos abstractos induyentes de todos los modos de apropiación de lo real, el cient́fico se especializa exdusivamente en las técnicas y procedimientos empleados en su práctica investigadora y en las prácticas de sus colegas que investigan objetos semejantes al suyo.

Esta "división del trabajo intelectual" conlleva múltiples limitaciones cognitivas. El alejamiento del epistemólogo de los procesos empíricos de construcción de conocimiento y de la sistematización de las técnicas y de los procedimientos usados en la investigación concreta, puede conduairlo a un criticismo paralizante de las prácticas constructoras de conocimiento científico que realizan otros investigadores, por la ausencia de propuestas investigativas prácticas. El epistemólogo percibe implicaciones lógicas de la práctica investigadora que generalmente el metodólogo y el cientúfico especialista disciplinario no logran percibir, pero el metodólogo posee un verdadero arsenal de técnicas y procedimientos que le permiten, rápidamente, escoger o construir instrumentos que hacen posible la percepción de formas y contenidos espeáficos de lo real. El especialista posee la experiencia de la utilización de múltiples técnicas y procedimientos en la construcción del conocimiento de algún aspecto de la teoría que ha asumido, en tanto que la reflexión epistemológica despojada de una práctica investigadora de objetos concretos conduce al desarrollo de constructos especulativos hipostasiados alejados de toda posibilidad práctica metodológica.

Es frecuente la producción de discursos teónicos ayyo único fundamento lo es un conjunto de obras leídas por sus autores. Se trata de construcciones teóricas totalmente especulativas en las que la fuente inspiradora se localiza en los contenidos de los disaursos sustantivos de obras leídas y no en los descubrimientos resultantes de un proceso riguroso de investigación cient́fica. Esto no significa ausencia de originalidad en los planteamientos; grandes teorizaciones han sido producto de este proceder pues, al conocer múltiples planteamientos, es posible construir reflexiones comprensivas y superadoras de sus fuentes generadoras si bien, en la mayoría de los casos, se acaba siendo un crítico de todo que nunca propone nada. Esta actitud es asumida muy frecuentemente por profesores de educación superior en el aula, que formulan grandes disertaciones "teóricas" verbales sin que se les conozca publicación alguna. Pero en los casos excepcionales en los que se lee para escribir y se escribe para que otros lean, se han construido aíticas demoledoras al método que fue empleado para construir un discurso sustantivo, sin haber realizado jamás investigación empírica alguna. En estos casos la conciencia se pasea por encima de entramados categórico-conceptuales, discursos sustantivos, datos empíicos y tratamientos estadísticos. 
La reflexión epistemológica exige una personalidad irreverente, hiperáítica y aguda que conduce, la mayoría de las veces, a la constitución de sujetos soberbios e intolerantes a las cuíticas de los demás. Abundan pues los críticos de todo y escasean los constructores de propuestas epistemológicas e interpretativas. El metodólogo, en cambio, es mucho más modesto que el epistemólogo pero, al igual que éste, casi siempre se encuentra muy alejado de las prácticas investigadoras de objetos concretos, por lo que se basa más en las explicaciones que dan los científicos de cómo investigan, que en experiencias personales al respecto. El problema que se genera es que los científicos no siempre reportan el procedimiento seguido como realmente se dio, no porque mientan deliberadamente, sino porque su falta de formación epistemológica no les permite identificar su filiación teórica y las implicaciones lógicas de su práctica investigadora. Por esto es por lo que, muchas de las veces, por partir de supuestos falsos se construyen grandes constructos metodológicos erróneos, como sucede en el caso del método hipotético-deductivo.

El estudio de las estructuras metodológicas requiere de personalidades sistematizantes, meticulosas y pulcras que conducen, mucha de las veces, a la constitución de sujetos apocados, mecanicistas, obsesionados por el procedimiento y el formato y con alcances intelectuales muy reducidos. El metodólogo muy freauentemente asume la práctica lectora de libros, artículos y reportes de investigación tan freaiente entre los epistemólogos, sólo que él se centra en los reportes de investigación científica y en los trabajados de métodos y técnicas de investigación elaborados por sus colegas. Esta centración en la sistematización de lo empírico de la práctica constructora de conocimiento, impide la percepción de la ubicación de esas prácticas en la lógica de la investigación cientúfica y su filiación a las grandes teorías. Podría tratarse induso, en algunos de los casos, de estar cerca de un gran descubrimiento pero, la centración intelectual en la práctica investigadora impide su percepción.

El cientúfico especializado sabe mucho de poco porque se centra en la construcción de conocimiento de un ámbito muy reducido pero buscando profundidad. Es frecuente el manejo de instrumentos, técnicas y procedimientos altamente sofisticados y el conocimiento profundo de las modalidades metodológicas aplicadas en su campo. Sobre todo entre los cientúficos de las ciencias físico-naturales, la especialización investigativa implica tanto la formación disciplinaria cientúfica en lo que a conocimiento generado se refiere, como a las técnicas, instrumentos y procedimientos utilizados en ese campo a los que han dado en llamar, incorrectamente, "metodologías". La centración en el conocimiento espećfico llega al detalle tanto en lo que a conocimiento generado se refiere, como a las maneras en las que fue generado. Sin embargo, la ausencia de una concepción totalizadora le impide la vinaulación de la lógica ontológica de su objeto con una concepción ontológica abstracta y de las técnicas y procedimientos de investigación utilizados por él, con una concepción epistemológica general. Todo esto impide el diseño de nuevas estrategias metodológicas y la construcción de teorizaciones con racionalidades distintas, colocando la práctica investigadora en la continuación permanente del trabajo de investigación en una sola racionalidad, cerrada a toda posibilidad de rupturas o incorporaciones de nuevas formas o contenidos ónticos y epistémicos. Lo ideal es formar sujetos dotados de conocimiento filosófico, epistemológico, metodológico y científico.

Explicar un método puede despojarlo de su esencia y presentarlo con una forma que le es ajena: la lógica de investigación es opuesta a la lógica de exposición porque la primera busca el conocimiento y, la segunda, dar a conocer lo conocido. Cuando lo conocido es la manera de conocer, la lógica de apropiación se transubstancia a lógica de exposición porque la substancialidad está constituida por las funciones de la razón y no por contenidos óntico-objetuales. A pesar de la contradictoriedad implicada en el intento de explicar el método, no existe ninguna posibilidad real de superar este problema. El objeto método tiene que ser explicado como proceso pues no existe otra manera de mostrar a otros de qué manera se realiza el proceso de construcción de teorizaciones. Sin embargo, para el científico especialista y para la mayońa de los metodólogos, la exposición de las técnicas y los procedimientos que se utilizan en los procesos de investigación no representa un gran problema ya que, como vimos, 
generalmente no se induye la reflexión epistemológica del proceso. Es el epistemólogo el que ha hecho consciencia de éste y otros serios problemas de los procesos de teorización.

Los efectos dañinos de la división del trabajo intelectual se pueden disminuir formando a los cientŕficos en epistemología, historia de la ciencia y metodología de la teoría asumida, e integrando la carga laboral de los académicos con actividades de investigación, docencia y difusión.

\section{La formación de científicos en el nuevo paradigma}

Los programas de formación profesional de filósofos y la formación filosófica en los planes de estudio de licenciatura y posgrado de las diferentes carreras profesionales, tienden a desaparecer. Se observa cómo durante los últimos años, de manera creciente, no sólo se tiende a considerar innecesaria la formación filosófica sino que se ha llegado a afirmar, induso, que lejos de coadyuvar a la formación profesional, distrae la atención y conduce a los sujetos a reflexiones dañinas en su ocupación laboral. La reflexión filosófica, se dice, sesga a los sujetos hacia preocupaciones intelectuales de carácter especulativo inútiles e improductivas, genera problemas existenciales y toma excesivamente críticos a los sujetos, auando lo que requiere la sociedad es individuos constituidos con características diametralmente opuestas a las que genera la refiexión filosófica. Y efectivamente, el régimen capitalista necesita individuos egoístas, pragmáticos, cosificados, consumistas, competidores, vanidosos, productores de objetos vendibles y obedientes; es decir, sujetos enajenados.

La escuela actual está organizada con base en la dívisión de todo. "El universo de la enseñanza de las ciencias es estrecho, descansa sobre la división. División de las ciencias y de las letras [...]; división de las ramas científicas [...]; división de los estudiantes entre sí; en fin, entre cada estudiante y él mismo: la ciencia enseñada es un universo cerrado en el que el estudiante no tiene ninguna participación verdadera, y que está completamente escindido de su vida concreta, de sus actividades cotidianas" (Badiou, 1980).

La supresión de la reflexión filosófica en la formación de los futuros científicos, va acompañada de la especialización cognitiva extrema. Dice Bachelard: "..el cientúfico es cada vez menos ávido de tales placeres totalitarios. Se ha repetido con frearencia que cada vez se especializa más. El filósofo, especialista en generalidades, se ofrece para las síntesis. Pero, de hecho, es a partir de una especialidad que el cientúfico quiere y busca la síntesis..." (2004). El conocimiento general es considerado también como un obstáculo para el conocimiento cient́fico porque la generalización inmoviliza el pensamiento y se ubica como un conocimiento vago que no tiene determinaciones precisas pues, al no detallar las particularidades del objeto o del fenómeno, se impide el análisis. El conocimiento especializado induye la presentación de la historia de la disciplina como un proceso continuo que ha desembocado en el ascenso a la verdad, encarmada en la teoría dominante en ese lugar y momento histórico. Esa historia es la historia de la cadena de teorías "equivocadas" que, con sus aportaciones parciales, finalmente dieron origen a la teoría actualmente hegemónica. No se trata de una historia del desarrollo de la ciencia como síntesis de la totalidad social, sino de una historia abstraída de las condiciones sociales, culturales y económicas, referida exdusivamente a los procedimientos de investigación, los instrumentos y las hipótesis.

A los futuros científicos no se les forma suficientemente en historia de la ciencia ni en epistemología. Al doctorante se le forma de acuerdo con las exigencias establecidas por el paradigma de una teoría asumida por el grupo que lo forma, proporcionándole sólo el conocimiento perteneciente a la teoría paradigmatizada. Esto conduce al desconocimiento de las condiciones histórico-sociales en las que cada teoría fue construida o en las que un descubrimiento científico fue realizado, no se conoce la estructura del conocimiento y no se sabe la problemática de la lógica con la que los diferentes conocimientos de un supuesto mismo objeto fueron construidos. Poseer una conciencia crítica, alta y reflexiva, también conduce a los científicos a lo impensado y a lo posible. 
Hoy día se estudia más para trabajar que para saber, pero esta teleología se contrapone con el espíritu científico. No hay duda de que existen conciencias teorizantes en el nivel más alto de cientificidad pero, para que la producción cient́fica se alejara totalmente del utilitarismo, es necesario la inexistencia de las clases sociales y que la sociedad haya llegado al nivel de satisfacción total de las necesidades, porque, en una sociedad dividida en dases, como lo es la capitalista, la producción de conocimiento teórico se da en conciencias cient́ficas purificadas. En la sociedad capitalista el conocimiento científico se transubstancia a tecnología y después a mercanća; la ciencia es fuerza productiva.

Se puede considerar que la formación de sujetos poseedores de conciencia teorizante implica tres ámbitos: el teórico-epistemológico, el teórico-disciplinario y el metodológico-investigativo. Por lo que se refiere al ámbito formativo teónico-epistemológico se observa en los programas de licenciatura de carácter práctico-utilitario, la dedicación de pocas asignaturas al análisis y discusión de los entramados categónico-conceptuales de los andamiajes teóricos más relevantes. En las licenciaturas de carácter investigativo la dosificación de contenidos es formalmente adeauada, pero el problema aparece en las estrategias didáctico-pedagógicas empleadas.

El fragmentarismo propio de la sociedad de consumidores actual aparece encamado en los planes de estudio de licenciatura como bloques formativos separados, conduciendo el abordaje de los contenidos teórico-epistemológicos como ajenos a los contenidos de los bloques teórico-disciplinario y metodológico-investigativo. El bloque teórico-disciplinario, además de que se imparte al margen de los otros dos, vive el problema, en las licenciaturas práctico-utilitarias, de que es abordado como un conjunto de saberes que debe poseer todo profesional de esa carrera, registrado en la memoria como rosario de definiciones y conocimientos producidos, al margen de sus estructuras categóricoconceptuales y, por tanto, de la lógica de investigación en la que se produjeron. El profesional sabe lo conocido en su dimensión aplicativa, pero no cómo se construyó ese conocimiento y, mucho menos, cómo se puede construir nuevo conocimiento.

La enseñanza del bloque metodológico-investigativo padece tres problemas principales: 1) El problema de su impartición al margen de los otros dos bloques, que ya fue tratado más arriba. 2) El problema de la enseñanza de la metodología al margen de la investigación. Este es uno de los problemas más agudos y. 3) El problema de la inexistencia de programas institucionales de investigación a los que se incorporen estudiantes y profesores.

En la mayoría de las instituciones de educación superior de nuestro país no se realiza investigación sino sólo docencia, por lo que la vida académica está centrada en la formación de profesionales eficientes y no de investigadores. En las instituciones que sí se realiza investigación de estado del conocimiento, aplicada y básica casi siempre se desarrollan proyectos independientes en los que participan pocos investigadores y uno que otro estudiante. Pero el problema no acaba aquí. En mudhas instituciones de educación superior en las que sí se realiza investigación, están diferenciadas las plazas académicas de los profesores de las de los investigadores, por ello evidentemente, son los investigadores los académicos más adecuados para impartir las asignaturas de carácter metodológicoinvestigativo.

La formación plena de científicos se concreta en posgrado y es ahí en donde las reglas de la "cientificidad" se encarnan en los planes de estudio, los programas de estudio de las asignaturas y las prácticas investigadoras. Para empezar, los posgrados forman sabios y no enuditos, no sólo se especializa y deshistoriza la ciencia, sino que se niega su enseñanza global pues el futuro cient́fico debe saber sólo de un fragmento de su disciplina, no la ciencia como totalidad ni tampoco las teorías espeáficas, dada la inconmensurabilidad entre ellas, por lo que debe alejarse de las generalidades y centrarse en el conocimiento concreto. 
Algunas medidas que podrían coadyuvar en la solución de este problema, son las modalidades de desarrollo de programas de posgrado aplicados principalmente en las ciencias físico-naturales y consistentes en integrar a los alumnos a los grupos de investigación. Es en estos grupos en donde el doctorante construye su objeto de investigación de tesis, analiza, expone y discute las fuentes de información de su objeto, experimenta y construye planteamientos.

Pero, si de lo que se trata es de alentar la formación de cient́ficos, es necesario entonces iniciar el proceso desde la educación básica, centrando la docencia en el aprendizaje de la lógica de las teorías científicas y no en la transmisión de los saberes generados por ellas, lo cual implica la formación de profesores con un perfil completamente diferente al existente. Para que un profesor sea estimulador del desarrollo del espíritu científico en sus alumnos, es necesario que cuente con una formación filosófica amplia, un conocimiento detallado de historia de la ciencia y un dominio de los andamiajes categónico-conceptuales de las diferentes teorías de un campo de conocimiento: las ciencias sociales, las matemáticas o las ciencias naturales.

\section{La conversión de los científicos al nuevo paradigma}

El científico es formado de conformidad con las exigencias de la teoría paradigmatizada, tanto en lo que se refiere al modelo de sujeto como a las condiciones en las que se realiza el proceso de formación profesional. Una de las primeras características a desarrollar en el estudiante es la del competidor. Al futuro científico se le hace competir con sus compañeros buscando derrotarlos, de modo que se habitúe a la vida que después llevará. "La preparatoria, y luego la facultad [...] no se apoyan en absoluto sobre el entusiasmo colectivo. Desarrollan, en cambio, el espíritu de competencia estrictamente individual, la astucia mediocre, el miedo al examen [...] La maquinaria escolar y universitaria quiebra poco a poco hasta el gusto por conocer, hasta el espíritu colectivo de lucha por el conocimiento" (Badiou, 2004).

El primer gran desafío de la formación de cientúficos es el de la transformación de la conciencia. En el ingreso a los programas de posgrado en ciencias, predominan alumnos poseedores de conciencias que operan bajo la forma empíica; "..no se trata, pues, de adquirir una cultura experimental, sino de cambiar una cultura experimental, de derribar los obstáculos amontonados por la vida cotidiana" (Bachelard, 2004). Dado que cada forma de la conciencia implica la operación con una lógica distinta a la de las demás y que lo que diferencia a la conciencia teorizante es la construcción de conceptos y el uso de categońas, resulta un verdadero problema hacer transitar de la lógica de la utilidad práctica a la lógica de la razón. Los estudiantes provenientes de licenciaturas investigativas están familiarizados con la sistematicidad y el proceder metódico en la construcción de conocimiento, por lo que el esfuerzo se centra en proporcionar conocimiento sustantivo y el herramental propio de la teoría asumida por el programa de posgrado y no en transformar la lógica con la que sus conciencias operan.

Al estudiante de ciencias se les transmite conocimiento sustantivo, categorías, reglas, pruebas, criterios y procedimientos, en un ambiente en el que su vida se desenvuelve en medio de salones de dase, auditorios, salas de cómputo, laboratorios, campos experimentales, discusiones cient́ficas, etcétera, de modo tal que poco a poco se va encarnando en él el mundo de la producción de conocimiento científico y tecnológico. "Sólo después de cierto número de esas transformaciones de la visión, el estudiante se convierte en habitante del mundo de los cient́ficos, ve lo que ven los científicos y responde en la misma forma que ellos. Sin embargo, el mundo al que entonces penetra el estudiante no queda fijo de una vez por todas, por una parte, por la naturaleza del medio ambiente y de la ciencia, por la otra. Más bien, es conjuntamente determinado por el medio ambiente y por la tradición particular de la ciencia normal que el estudiante se ha preparado a seguir" (Kuhn, 1986). 
La comunidad científica que está formando al alumno, le hace leer los libros y los artículos que considera convenientes porque contienen el "conocimiento objetivo de la disciplina", es decir, los textos construidos desde la teoría de la cual se participa. El centro de la formación de científicos está en el método de una teoría. No se enseñan los métodos utilizados por las diferentes teorías generadas en el campo disciplinario, sino el método de la teoría hegemónica en la comunidad cientŕfica en la que se está dando el proceso de formación. La vida cotidiana en los centros e institutos de investigación acaba por encamarse en los sujetos y la teoría paradigmatizada acaba siendo racionalidad de las conciencias, objetos e instrumentos de investigación, hipótesis, diseño de laboratorios e instalaciones y proyectos existenciales individuales.

¿Qué hacer? La producción científica asume las formas de la sociedad en la que existe. Cualquier medida "correctiva" que se quiera introducir en la dinámica del sistema acabará desechada o integrada al mismo, ajustada a la racionalidad de ese sistema. La lucha por la transformación de la ciencia es una lucha por la transformación de la sociedad, mas no se debe suponer que la lucha en el interior de la ciencia, por sí sola, puede transformar a la sociedad entera, ni que la lucha por la transformación de la sociedad tenga que darse por fuera de la ciencia $y$, una vez realizada, ser llevada a su interior. Dice Feyerabend: "...debemos impedir que los cient́ficos tengan la responsabilidad de la educación y que enseñen como un 'hecho' y como el 'único método verdadero' aralquier cosa que nos depare el mito de tumo" (Feyerabend, 2003).

La conversión a la nueva teoría pareciera colocar al cient́fico ante un mundo nuevo. "Guiados por un nuevo paradigma, los cient́ficos adoptan nuevos instrumentos y buscan en lugares nuevos. Lo que es todavía más importante, durante las revoluciones los cientŕicos ven cosas nuevas y diferentes al mirar con instrumentos conocidos y en lugares en los que habían buscado antes" (Kuhn, 1986: 176). Pero no sucede así entre todos los científicos. Aquellos ayos éxitos científicos fueron logrados en un paradigma en desgracia, desprecian las nuevas teońas. La profesionalización cientúfica paradigmática conlleva el que se asuma una postura dogmática férrea pues, entre mayor es la especialización investigativa, menor es la posibilidad de pensar problemas no considerados por el paradigma. "Por una parte, esta profesionalización conduce a una inmensa limitación de la visión de los cientúficos y a una resistencia considerable al cambio del paradigma. La ciencia se hace así cada vez más rígida" (Kuhn, 1986).

Lo cierto es que una vez que una teońa es considerada como "explicación adecuada" de una anomalía, se inicia un proceso de conversión de los científicos y de las instituciones adoptando las condiciones planteadas por ella. $Y$ uno de los mayores impulsores de la conversión es el financiamiento de la investigación científica y las condiciones de conservación y elevación de la jerarquía como científico. Cuando una institución académica asume una teoría como paradigma, obliga a los solicitantes de financiamiento a presentar los proyectos de investigación y los reportes de resultados en el formato implicado en esa teoría, de modo tal que, no ajustarse al nuevo paradigma puede significar el quedar fuera de la posibilidad de continuar profesionalmente dedicado a la investigación.

En algunos centros de investigación toda la comunidad participa de una sola teoría; en otros, coexisten dos o más teorías, pero una de ellas es la hegemónica. Asumir una u otra hace la diferencia entre contar con apoyo para la investigación, la asistencia a reuniones cientúficas, publicar, etcétera y quedar en el ostracismo. Induso, muy frecuentemente, las revistas científicas sólo publican aquellos trabajos que embonan con su paradigma, así como los comités revisores de ponencias para reuniones cient́ficas. También contribuye el aparato gubernamental y la dirección empresarial en este proceso de paradigmatización de la ciencia. La asesońa a empresarios y políticos se realiza a través de proyectos vinculados con las necesidades de desarrollo empresarial, con la solución de problemas sociales políticamente relevantes y con la difusión de explicaciones supuestamente cient́ficas que generen estados de conciencia deseados de las masas, de conformidad con los intereses de los financiadores. Dice Habermas al respecto: "... a nivel de 
gobiemo han sido instituidas burocracias encargadas de dirigir la investigación y el desarrollo e institutos de asesoramiento cient́fico, ayyas funciones reflejan una vez más la peculiar dialéctica de la transformación de la ciencia en práctica política" (Habermas, 1999).

La formación en el terreno de la epistemología y la historia de la ciencia, señalada al final de la primera parte de este escrito, ampliaría los horizontes de teorización y operaría una apertura de la razón a teorizaciones sustentadas en diferentes racionalidades.

\section{Conclusiones}

$>$ Los procesos de paradigmatización de una teoría conllevan la formación de cientúficos en esa teoría. Formar científicos en una teoría significa convertir las concepciones ontoepistemológicas de esa teoría en la manera en la que el futuro cientúfico piensa que es la realidad y la manera de construir su conocimiento.

$>$ La fragmentación pedagógica del proceso de formación de científicos obstaculiza la constitución de concepciones integrales de la ciencia, amén de que en la educación básica, media, media superior y superior el conocimiento cient́fico es enseñado como saberes, la historia de la ciencia como acumulación de conocimiento y lucha entre la verdad y la mentira.

> La formación paradigmática de científicos desprecia la historia de la ciencia en general y de la disciplina espeć́fica en particular, así como el entendimiento de las racionalidades de las diferentes teorías que constituyen el campo de dominio de su disciplina.

$>$ El cient́fico es formado ayuno de filosofía en general y de filosofía de la ciencia en particular, pues los cientúficos consolidados que lo son de una teoría, asumen la racionalidad de ella como metarracionalidad y como punto de llegada de toda la historia de la ciencia. El pasado de la ciencia no es más que el camino para llegar a la verdad epistemológica encamada en la teoría paradigmatizada.

$>$ Los enigmas de la teoría paradigmatizada son los objetos de investigación de la ciencia. Los demás objetos son metafísicos.

$>$ Las condiciones en las que se forman académicamente los cient́ficos, es decir, los planes de estudio, los programas de estudio de las asignaturas, los laboratorios, los campos experimentales, los instrumentos, las pruebas de verificación, etcétera son tales que garantizan la reproducción puntual de los formadores y la conservación de la hegemonía de la teoría en cuestión.

> Es hasta que los representantes de una comunidad cientíica, que en realidad opera como comunidad epistémico, está convencido de que el estudiante es una encarnación de la teoría paradigmática que se le otorga el grado de doctor, lo cual significa que ya es miembro de esa comunidad.

> Las diferentes teorías no son más que perspectivas cognitivas diferenciales de lo real, que permiten percibir formas y contenidos espećficos intraducibles entre sí por participar de racionalidades diferentes también.

$>$ El profesor formador de científicos debe ser científico y poseer una formación sólida en epistemología, historia de la ciencia en general, historia de las teorías asociadas a su línea de investigación y en la teoría desde la cual realiza su práctica investigadora. 


\section{Bibliografía}

Bachelard, G. (2004). La formación del espíritu científico. México: Siglo XXI

Badiou, A. (1980). ¿Qué es hoy un estudiante de ciencias? En Levy-Leblond, J.M. y A. Yaubert. (Auto) crítica de la ciencia (pp. 213-220). México: Nueva Imagen.

Feyerabend, P. (2003). Tratado contra el métoda. Madrid: Tecnos.

Habermas, J. (1999). Ciencia y técnica como "ideología". Madrid: Tecnos.

Kuhn, T. S. (1986). La estructura de las revoluciones científicas. México: FCE. 